\title{
Simultaneous power quality analysis of feeders in MV utility power stations
}

\author{
Aleksandar Nikolic, Blagoje Babic, Aleksandar Zigic, Nikola Miladinovic, Srdjan Milosavljevic \\ Electrical Engineering Institute Nikola Tesla, University of Belgrade, Koste Glavinica 8a, 11000 Belgrade, Serbia
}

\section{ABSTRACT}

The development of a low-cost, space saving device that could simultaneously take measurements from all (usually eight or up to twelve) outgoing three-phase feeders in a distribution substation is presented in this paper. To meet these requirements, at least 3 voltage measurements and 36 current measurements should be performed the same time. In order to save space but not to reduce the measurement accuracy, a data acquisition system is designed based on real-time multiprocessing with a microcontroller and an FPGA circuit. Voltage and current measurements and their corresponding higher-order harmonics are calculated using a fast FPGA circuit, while other calculations (power, power factor, voltage and current phase angles, etc.) are performed in the microcontroller. Further savings are obtained using multichannel analog input modules with multiplexed inputs. Communication with the supervising computer is done using a GPRS modem or wireless network module depending of the station location. Results obtained in the laboratory and later in an industrial prototype confirm the proposed solution.

\section{Section: RESEARCH PAPER}

Keywords: Power Quality; Simultaneous measurements; Analysis; Utility Power Station

Citation: Aleksandar Nikolic, Blagoje Babic, Aleksandar Zigic, Nikola Miladinovic, Srdjan Milosavljevic, Simultaneous Power Quality Analysis of Feeders in MV Utility Power Stations, Acta IMEKO, vol. 4, no. 1, article 9, February 2015, identifier: IMEKO-ACTA-04 (2015)-01-09

Editor: Paolo Carbone, University of Perugia

Received December $15^{\text {th }}, 2013$; In final form December $11^{\text {th }}, 2014$; Published February 2015

Copyright: (C) 2014 IMEKO. This is an open-access article distributed under the terms of the Creative Commons Attribution 3.0 License, which permits unrestricted use, distribution, and reproduction in any medium, provided the original author and source are credited

Funding: (none reported)

Corresponding author: Aleksandar Nikolic, e-mail: anikolic@ieent.org

\section{INTRODUCTION}

The increased demands for electricity created extensive power generation and distribution grids. Industries demand larger and larger shares of the generated power, which, along with the growing use of electricity in the residential sector, stretched electricity generation to the limit.

Today, electrical utilities are no longer independently operated entities; they are part of a large network of utilities tied together in a complex grid. The combination of these factors has created electrical systems requiring power quality [1],[2].

Contemporary concepts of measuring instruments for monitoring power quality are based on sophisticated solutions in the field of information technology with advantages in terms of reliability, conformity, speed of operation, automatic control and management of quality on the principles of distributed intelligent measurement methods using software tools, especially virtual instrumentation.
This also becomes important for smart grid solutions, where decisions for different actions in the local network are also based on the power quality analysis [3]-[6].

\section{REQUIREMENTS FOR DATA ACQUISITION EQUIPMENT}

The choice of components and equipment in the preparation of the conceptual design of the measuring system which function is to measure and analyse the power quality is defined by the needs of distribution companies [7], [8]. Given the needs of distribution substations where the usual numbers of feeders to consumers are 8 or 12 , a solution should be found that will allow simultaneous measurements up to 12 feeders. In this way, in the case of an analysis of the impact of changes in consumer power quality on the part of the network that is powered from a substation can unambiguously determines the feeder with the problem. Another limiting factor is typical device dimensions. The device shall be mounted on the wall inside the building substation or pole-mounted transformer with 


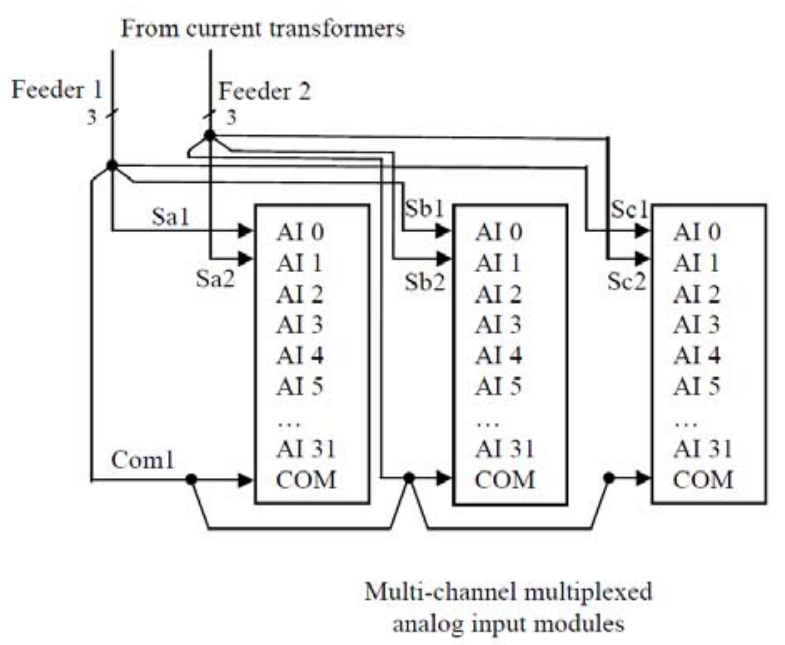

Figure 1. Proposed measurement principle.

pole. Also, to provide mobility, i.e. portability, the device should be as small as possible just in case that it is used in a number of substations. Some problems that could arise are summarized hereafter.

The necessity to set some parameters with a frequency at least $10 \mathrm{kHz}$ in order to better register voltage and current waveforms, and thus the potential disturbances to the sine waveform, requires that acquisition software should measure values in real time. Additionally, because of the need to apply the discrete Fourier transform (DFT) to calculate the content of higher harmonics [9] of voltage and current of up to the $50^{\text {th }}$ order, programmable FPGA circuits and their flexible reprogramming that enables the use of rapid calculations on a hardware level are considered [10],[11].

Three phase measurements without phase delay between phases of the same feeder, as a consequence of multiplexing several analogue inputs using one $\mathrm{A} / \mathrm{D}$ converter, are achieved by connecting each phase of one feeder to a different input module. Since measurements on all modules can be initiated in the same time without delay, all three phase currents of one feeder can be measured without phase delay between phases. The phase delay between adjacent phases as an effect of the multiplexer is lower than the acceptable accuracy level. Since this phase delay is constant, it could be additionally compensated in software if higher accuracy is requested. The proposed measuring principle is shown in Figure 1, where AI denotes analogue inputs $( \pm 500 \mathrm{mV}$ level$)$ on 32 channel modules connected on the same data communication bus. For simplicity, only measurements on two feeders are shown. Signals from further feeders (up to 32) are connected in the same manner.

The measurement range is selected based on the output of split core current transformers that have $333 \mathrm{mV}$ rated output. Such transformers are chosen in order to have a simple installation in the field with an opportunity to move the acquisition system to another power station. Also, $\mathrm{mV}$ output gives the possibility to directly feed signals to the acquisition system without the need for additional signal

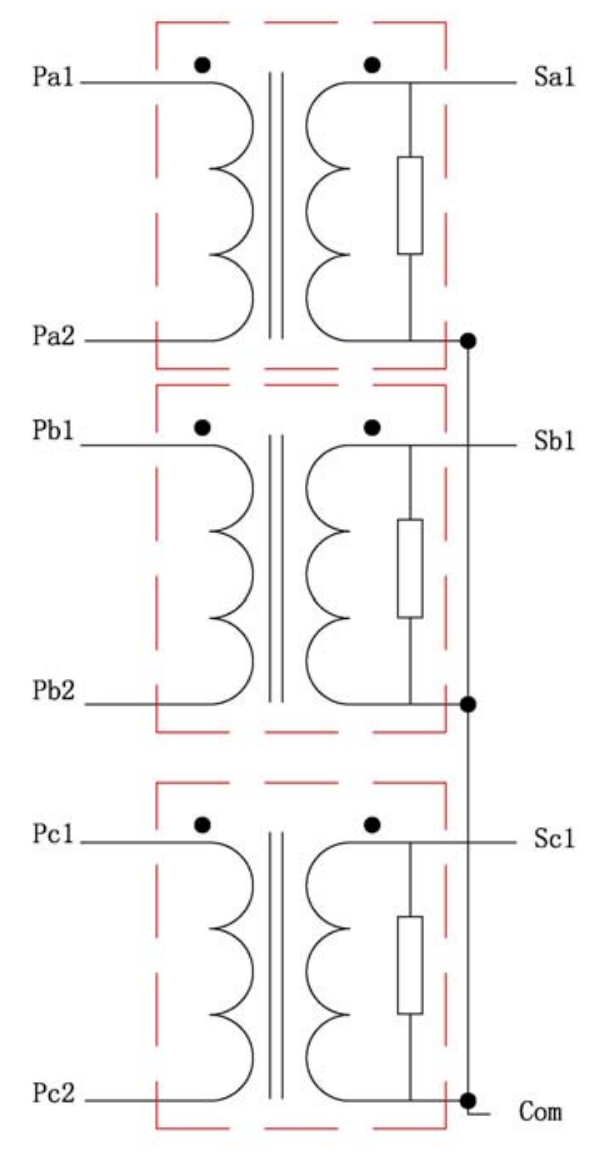

Figure 2. Connection of split core current transformers with voltage output.

conditioning. A way of connecting current transformers on a three-phase feeder is shown in Figure 2. It can be observed that the same common line on one three-phase feeder additionaly reduces the overal number of connections, what is a merit for the industrial implementation.

\section{PROTOTYPE DEVELOPMENT}

\subsection{Laboratory prototype}

In order to check the functionality and accuracy of the system, at first a laboratory prototype is developed. It is based on a data acquisition USB card and electronics cards that simulate the three-phase system [10]. This specially developed card has two inputs connected to the USB data acquisition card used for simulating voltage and current. In order to have three voltage and three current signals at the output, the card uses phase shifters to provide additional signals with $120^{\circ}$ phase difference. This card needs external power supply of \pm 15 VDC.

The proposed multi-channel power quality acquisition system is built up using a programmable controller NI cRIO 9076 with 4 modules, one 230 VAC three channel voltage measurement module and three 32 channel voltage analogue inputs modules. The same controller configuration is used for the industrial prototype.

Figure 3 shows the complete laboratory setup. The electronics card that emulates the three phase system is placed on top of the DC power supply. The outputs of the 


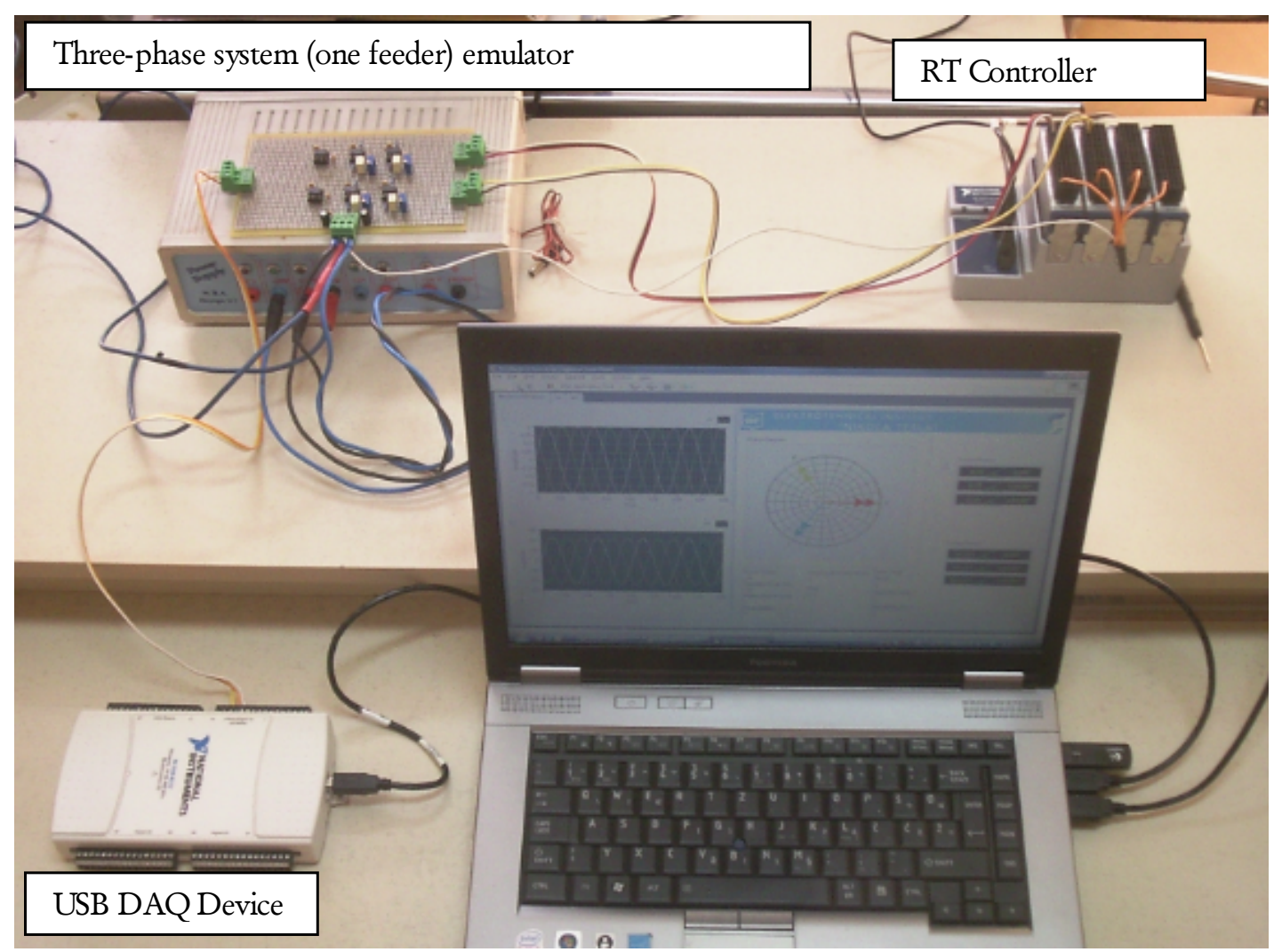

Figure 3. Laboratory prototype.

emulator are 6 voltages of $\pm 100 \mathrm{mV}$, where three signals are representing feeder voltages of phases $R, S, T$ and the

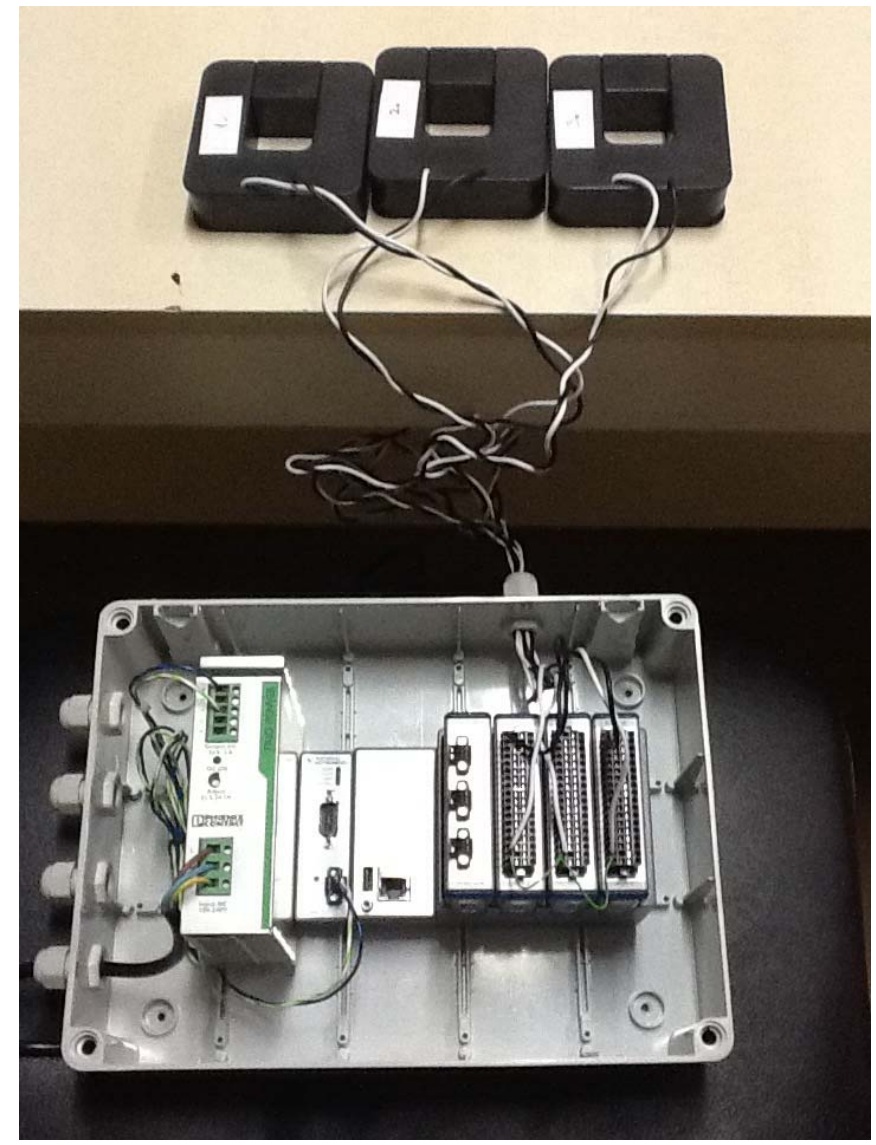

Figure 4. Industrial prototype with one feeder current measurements. other three signals are currents of the corresponding phases.

\subsection{Industrial prototype}

The advantage of the hardware-in-the-loop (HIL) approach [10], used for the laboratory prototype shown in the previous chapter, is a shorter developing time for the final solution. This relies on the real-time controller, since it is used both in the laboratory and the industrial prototype. Furthermore, a real-time algorithm running on the microcontroller and the FPGA is retained for the industrial prototype and only changes were made regarding the principle of voltage measurement.

In order to further reduce the number of requested measurements, one three-phase voltage is measured using the three channel module with isolated 300 VAC inputs. Such approach is possible due to the fact that in a utility power station all feeders are supplied from one or more power transformers connected to the same high voltage

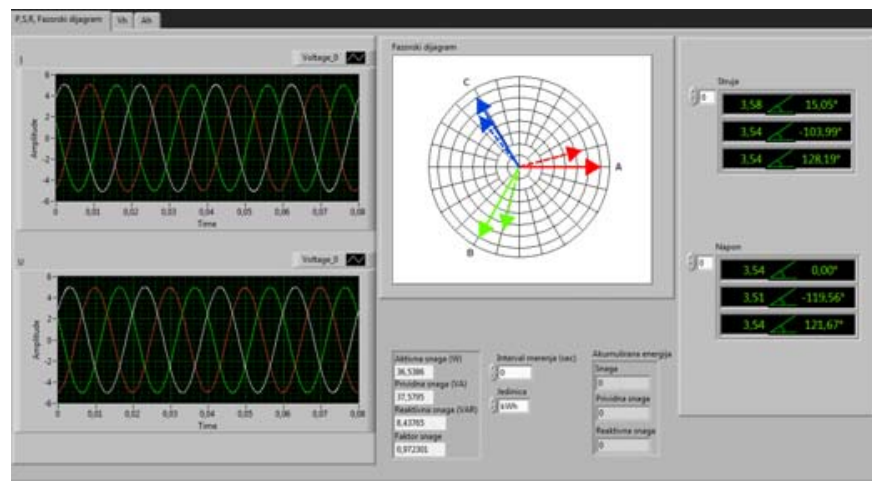

Figure 5. First part of real-time application. 


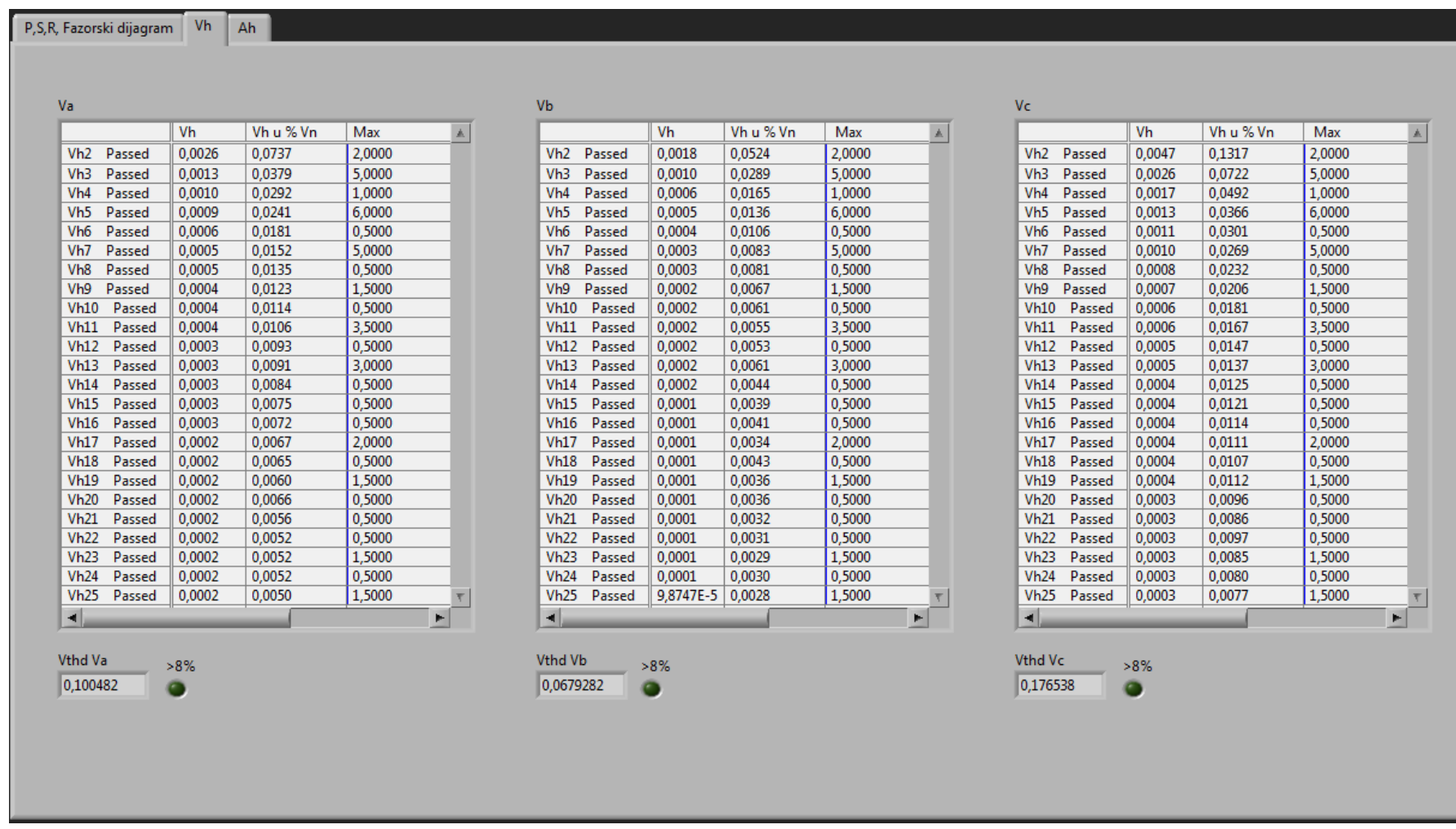

Figure 6. Voltage high-order harmonics.

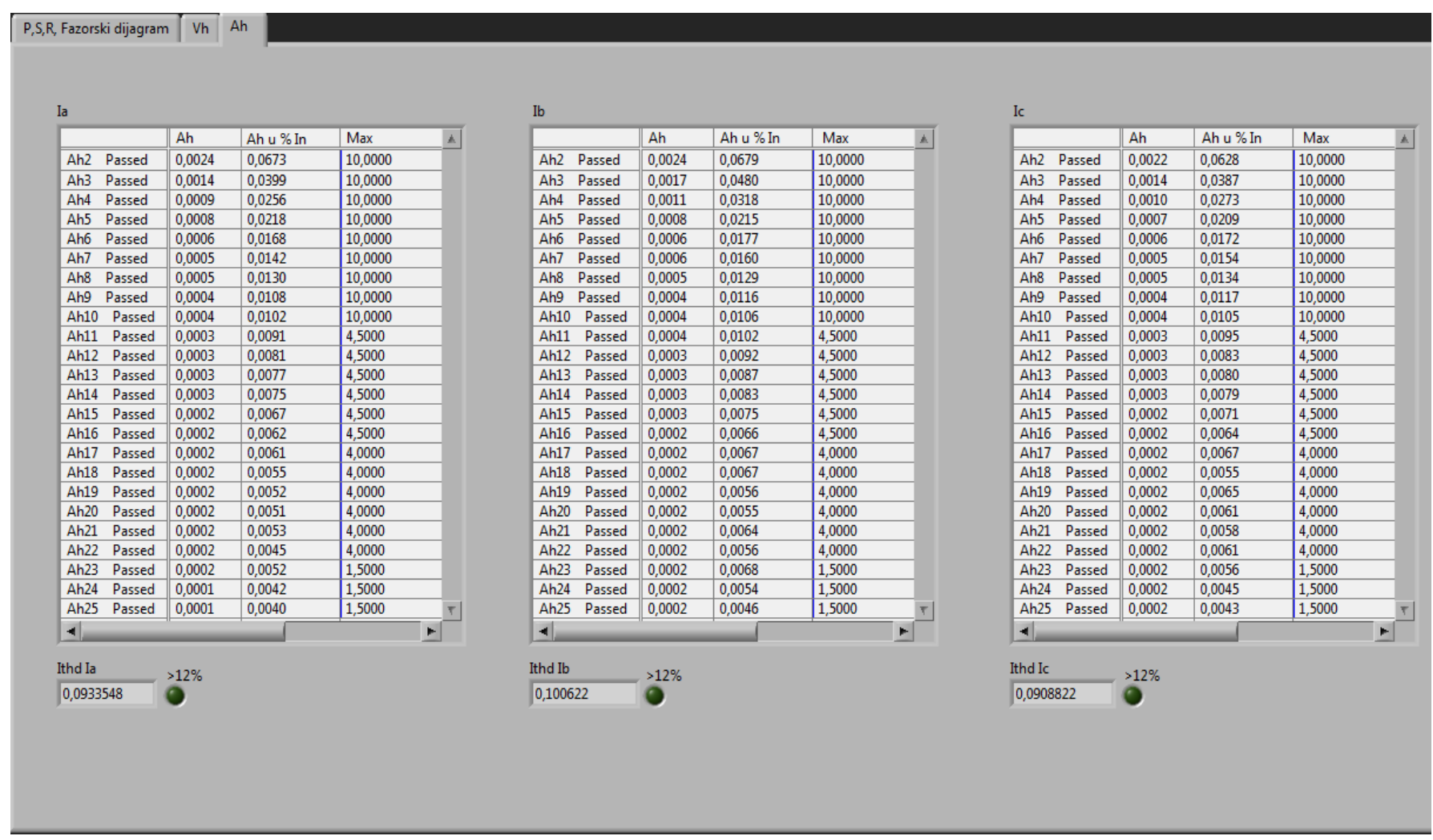

Figure 7. Current high-order harmonics.

line.

The difference between laboratory and industrial tests is in the signal introduced to the controller to emulate the three-phase voltage in case of the laboratory prototype. For these tests the same analogue input module used for current measurements is also used for voltage measurement. On the other side, in the industrial prototype real phase voltages of 230 VAC are introduced. For that module, initialization and calculations are different. But, that doesn't influence the other parts of the real-time software, especially higherorder harmonics calculations performed on hardware level using the FPGA. 


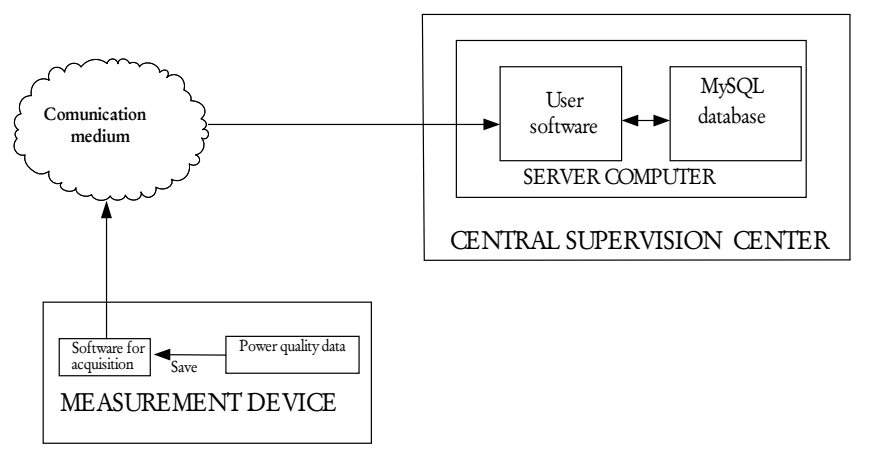

Figure 8. Concept of proposed power quality system.

In Figure 4 an industrial prototype is shown in opened housing. For communication to the supervisory computer Ethernet is used or a GPRS modem connected to the RS232 port.

The controller also has a USB port for external USB memory sticks or even a USB hard disk. In that case a large amount of data could be saved without the necessity for real-time data transfer to another computer. That could be also a merit in cases where the Ethernet network is not available or mobile signal strength is poor for the GPRS modem.

\section{DEVELOPED SOFTWARE}

In order to use all features of the proposed real-time controller, like FPGA for computationally high intensive algorithms [11], and at the same time visualisation on a client computer, software is coded using thr graphical programming language LabVIEW, a software development environment that contains numerous components, several of which are required for any type of test, measurement, or control application [12],[13].

\subsection{Real-time application}

The real-time application, developed in LabVIEW and built with Real-time and FPGA options, consists of three parts and it is the same for both the laboratory and the industrial prototype. This approach provides the possibility to test every aspect that could occur in the real system, while reducing time for final tuning of the software.

The first part of the application shows measured or simulated input voltages and currents per feeder, phasor diagram, phase angles and calculated power (active, reactive and apparent) and power factor. It is also possible to get accumulated values (Power Usage) in Wh/kWh/Wh. This part of the application is shown in Figure 5.

The second and third parts of the application are indicated as $\mathrm{Vh}$ and $\mathrm{Ah}$, respectively. Both parts are devoted to high-order harmonics of voltages $(\mathrm{Vh})$ and currents (Ah) shown in the tables, with a clear indication if values exceeding limits defined in the standards [9], [14]. Also, the total harmonic distortion (THD) is shown both for voltage and current, with a colour signal indicating values higher than the limits ( $8 \%$ for voltage and $12 \%$ for current). These two parts of the application are shown in Figures 6 and 7.

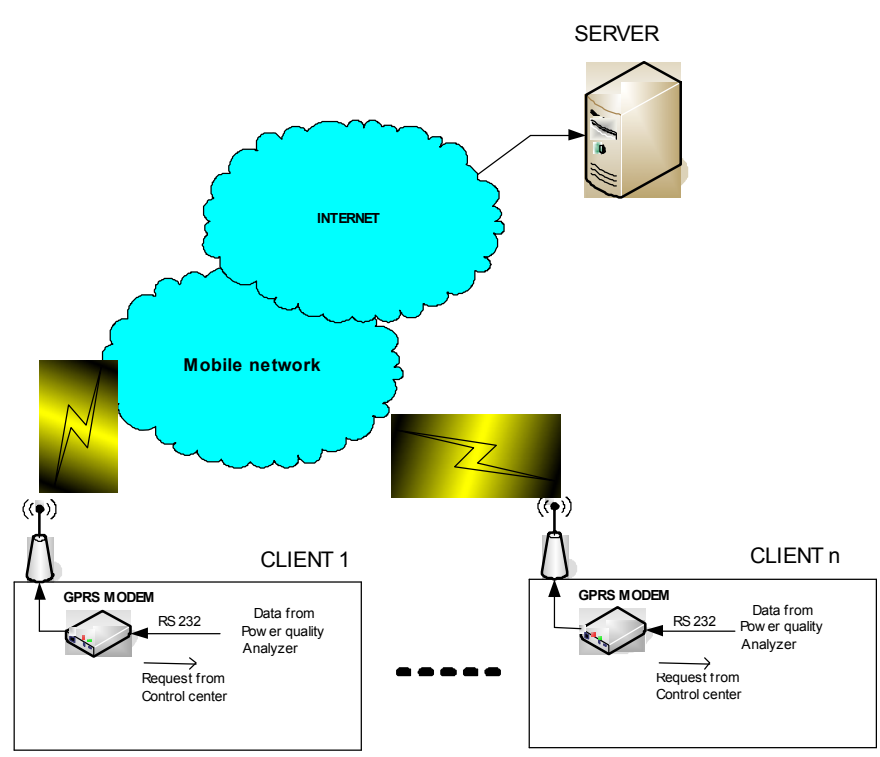

Figure 9. Communication interface using GPRS protocol.

\subsection{Application for data collecting and analysis}

In order to centralize power quality measurements of different consumers, it is required to develop a dedicated database and client application in addition to making the measuring device [15]. The role of the database is to allow storage of measured data, while a client application collects data from the data acquisition software through an appropriate communication medium for the selected protocol. The roles of client applications are to perform the display of archived data and perform when signal processing is needed. The concept of a system that includes a database client application and a power quality measuring device is shown in Figure 8.

\subsubsection{Database structure}

The main purpose of the database is to collect the data important for further power quality analysis of supplied electricity. The database is organized regarding to the objects and relations in the power system, but in such a manner to satisfy specific user requirements. Modular database organization enables its extension to other power objects and consumers. The database also uses auxiliary tables, like standard limits, measurement methods and instrument technical characteristics. As a system for database control, MySQL 5.1 is used. Due to the advanced features, the InnoDB engine has been chosen.

\subsubsection{Client application structure}

For client application development, Visual C\# is used as a programming language, .NET development environment and Visual Studio as a development tool. Development environment .NET has a library of base classes for access to different databases, work with XML files, Web and Windows forms, and connection through Remoting and Web services. COM (Component Object Model), Word and Excel automatization are also used because most customers like to perform additional calculations in Excel and to use Word as a report creating tool. 


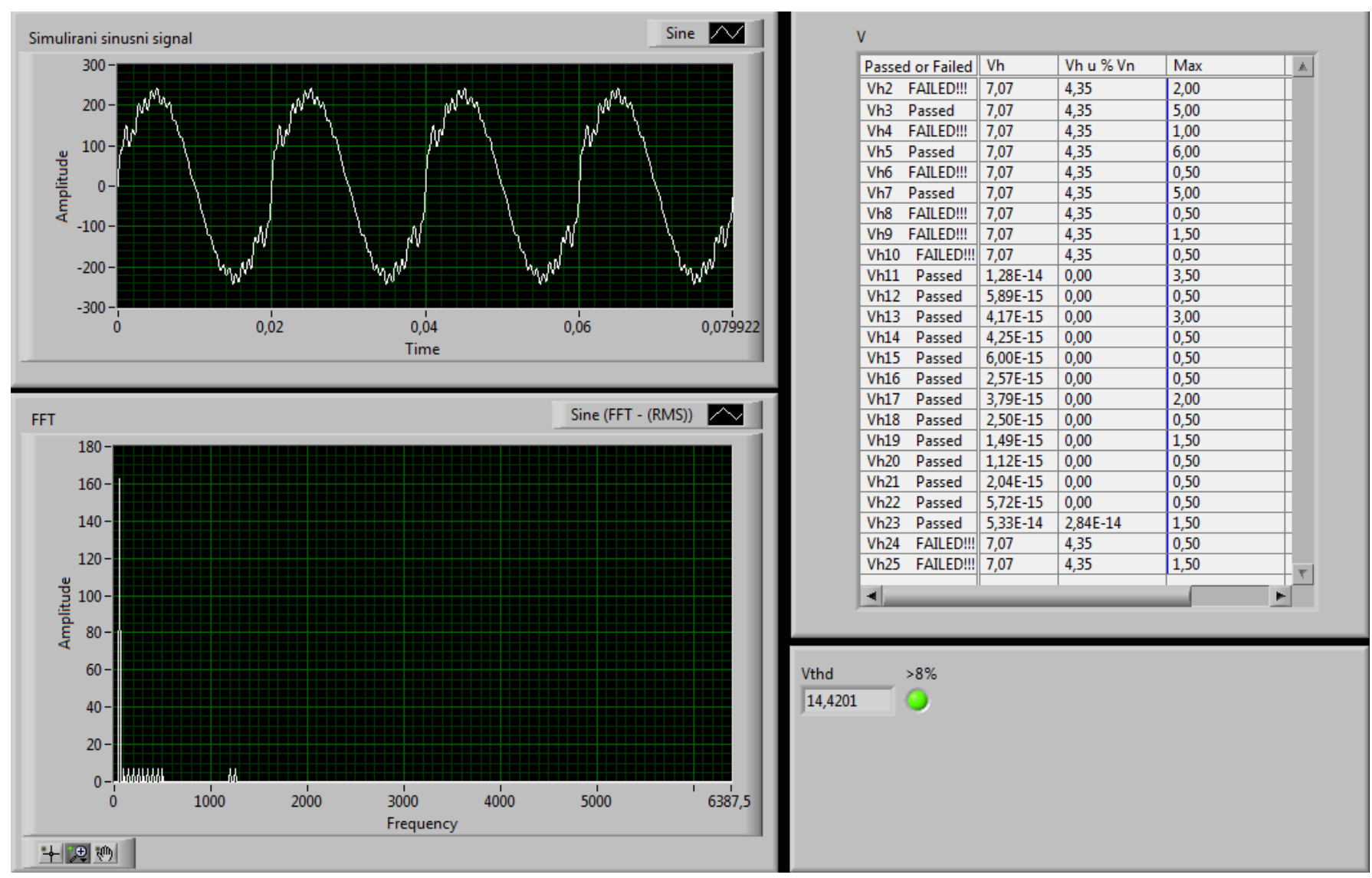

Figure 10. Measurement results of a test signal with superimposed harmonics.

\subsubsection{Data transmission from measurement devices}

In order to transfer data from the measurement device to the client computer, mostly located in the control centre of the local utility, a different communication medium can be used.

After an analysis of different interfaces and protocols [16]-[18], two solutions for communication between local measurement devices mounted in the utility power station and control/diagnostic centre are chosen. The first one is Ethernet, for locations that exist in or is nearby a local computer network and a GPRS modem for longer distances and for instance pole mounted power transformers. As an upgrade of Ethernet, digital radio modems over DNP3 protocol and Wireless LAN can be used.

The protocol Modbus is proposed in two versions: Modbus RTU and Modbus TCP/IP. Modbus RTU is used for communication between the metering and the data acquisition device and the local modem (usually GPRS), while the modem provides direct translation of data to TCP/IP. In this way it enables further uniformity of data transfer, regardless of whether it uses a GPRS wireless interface or the classic Ethernet network. Also, it is suitable for networking computers to exchange data retrieved from the local measuring points [19].

Figure 9 shows a configuration of a communication system with GPRS implemented protocol with client-server architecture.

\section{EXPERIMENTAL RESULTS}

\subsection{Laboratory tests}

In order to check the proposed multichannel power quality measurement system, first a test signal of 230VAC $50 \mathrm{~Hz}$ is used (pure sine signal). In that case we could easily verify correct calculations in software. Next,a test signal is superimposed with harmonics $(100,150,200,250,300,350$, $400,450,500,1200$ and $1250 \mathrm{~Hz}$ ) with an amplitude of 10 $\mathrm{V}$. The obtained signal is shown in Figure 10. It can be observed that total harmonics distortion is higher than the permissive value from the standard [14]. On the bottom diagram a Fast-Furrier transformation of the signal is shown. Several values of higher-order harmonics that exceed limits are indicated as FAILED in the table shown in Figure 10.

The results show correctness of the proposed methods for power quality analysis [20], [21]. Also, the part of the system based on FPGA provides fast, real-time calculations on several channels.

\subsection{Criteria for selecting typical measurement points}

Prior to putting the measuring device in an industrial environment, typical measurement points should be selected. One criterion is higher-order harmonics content, since the electricity usage is related to the expected current or voltage distortion in the distribution network. 


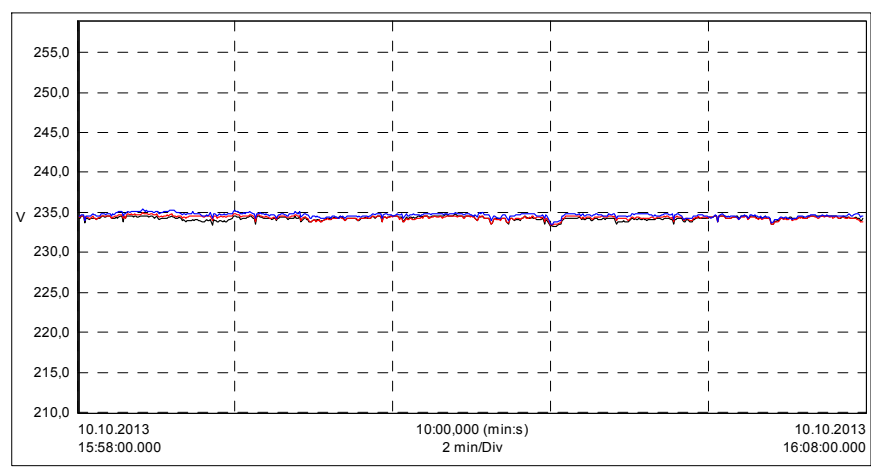

Figure 11. Phase voltage trend.

Criteria of expected higher-order harmonics are defined by the index of expected higher-order harmonics content, calculated for every power station X/0.4 k:

$$
\mathrm{IH}_{\mathrm{i}}=\sum_{\mathrm{j}=1}^{5} \mathrm{k}_{\mathrm{Hj}} \cdot \frac{\mathrm{W}_{\mathrm{Aj}, \mathrm{i}}}{\mathrm{W}_{\mathrm{Ai}}}
$$

where $\mathrm{kHj}$ is the weighted coefficient of power consumption of consumers' group $j, W_{A j, i}$ is the active energy of the consumers group ,j" according to the categorization based on higher-order harmonics and supplied from power station $\mathrm{X} / 0.4 \mathrm{kV}$ "i”, and

$\mathrm{W}_{\mathrm{Ai}}$ is the active energy of all the consumers supplied from power station $\mathrm{X} / 0.4 \mathrm{kV}$ “i”.

The power station with the highest value of index $\mathrm{IH}_{\mathrm{i}}$ is the first candidate for placing the power quality supervision system.

\subsection{Testing of the industrial prototype}

Using previously defined criteria, as a consumer for testing the industrial prototype an office building is used. Such buildings are usually equipped with a lot of computers, air-conditions and fluorescent light. In that case, large office buildings are consumers with high value of total harmonic distortion (THD).

In the following Figures some results taken from a main supply cabinet in one office building are presented.

Figure 11 shows the phase voltage RMS trend, Figure 12 the voltage total harmonic distortion, while Figure 13 presents the total harmonic distortion factor of current.

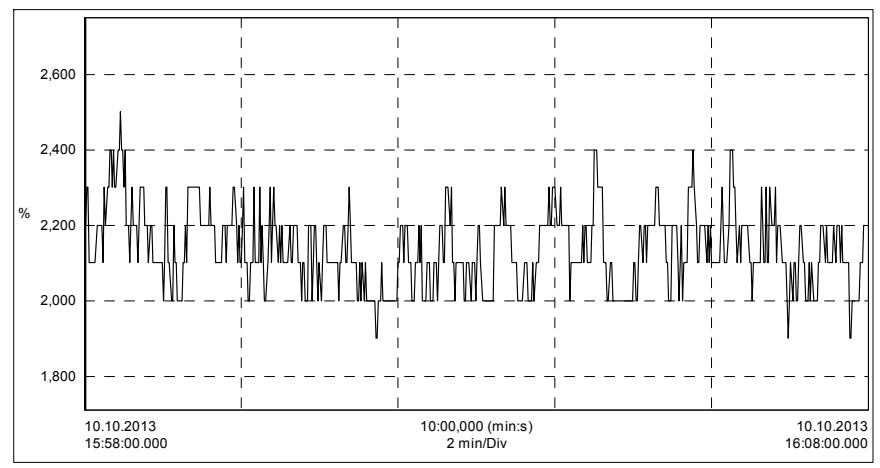

Figure 12. Voltage total harmonic distortion trend.

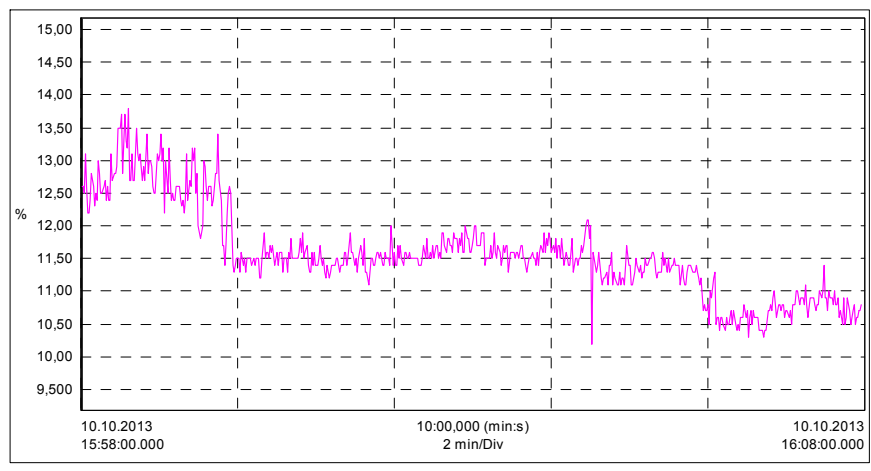

Figure 13. Current total harmonic distortion trend.

\section{CONCLUSIONS}

One solution for solving requirements for simultaneous multi-channel power quality measurement in distribution networks is presented in this paper. It is shown that using state-of-art FPGA technology and properly configuring real-time software computationally intensive tasks like DFT of signals with harmonics up to $50^{\text {th }}$ can be solved. This enables power quality analysis on 12 channels simultaneously, what is a merit of the proposed system and also requirements by the distributor operator is fulfilled.

Furthermore, with the proposed measurement arrangement it is possible to measure simultaneously up to 32 feeders or 96 currents along with 3 voltages, while device dimensions and weight are still less than required up to $500 \times 500 \times 200 \mathrm{~mm}(\mathrm{WxHxD})$ and $5 \mathrm{~kg}$. In this case mobility requirements for the measuring system are also retained.

The proposed solution has also reduced developing time. A programmable controller, introduced in the first development stage for the laboratory prototype is used with the same real-time software as in the industrial prototype, thanks to the HIL concept applied during laboratory tests.

Results obtained from both development phases show good correlations.

\section{REFERENCES}

[1] C. Sankaran, Power Quality, CRC Press LLC, Florida, 2002.

[2] Kilter, J.; Meyer, J.; Howe, B.; Zavoda, F.; Tenti, L.; Milanovic, J.V.; Bollen, M.; Ribeiro, P.F.; Doyle, P.; Romero Gordon, J.M., "Current practice and future challenges for power quality monitoring - CIGRE WG C4.112 perspective," 2012 IEEE 15th International Conference on Harmonics and Quality of Power (ICHQP), , pp.390,397, 17-20 June 2012.

[3] Meyer, J.; Kilter, J.; Howe, B.; Zavoda, F.; Tenti, L.; Romero Gordon, J.M.; Milanovic, J.V., "Contemporary and future aspects of cost effective power quality monitoring Position paper of CIGRE WG C4.112," Electric Power Quality and Supply Reliability Conference (PQ), 2012 , pp.1,6, 11-13 June 2012.

[4] Music, M.; Bosovic, A.; Hasanspahic, N.; Avdakovic, S.; Becirovic, E., "Integrated Power Quality Monitoring Systems in smart distribution grids," 2012 IEEE International Energy Conference and Exhibition (ENERGYCON), pp.501,506, 9-12 Sept. 2012. 
[5] Laskar, S.H.; Khan, S.; Mohibullah, "Power quality monitoring in sustainable energy systems," Sustainable Systems and Technology (ISSST), 2012 IEEE International Symposium on, pp.1,6, 16-18 May 2012.

[6] Cornoiu, M.; Barbulescu, C.; Jigoria-oprea, D.; Kilyeni, S.; Prostean, G.; Teslovan, R., "Power quality monitoring and analysis. Case study for 220/110 kV substation," 2011 IEEE 3rd International Symposium on Exploitation of Renewable Energy Sources (EXPRES), pp.133,138, 11-12 March 2011.

[7] Chen, S.; Zhang, C.L.; Liu, Y.Z., "A multi-channel monitoring system for system-wide power quality measurements," Power System Technology, 2000. Proceedings. PowerCon 2000. International Conference on, vol.2, pp.953-958 vol.2, 2000.

[8] Ruiz-Llata, M.; Guarnizo, G.; Boya, C., "Embedded power quality monitoring system based on Independent Component Analysis and SVMs," The 2011 International Joint Conference on Neural Networks (IJCNN), pp.2229,2234, July 31 2011-Aug. 52011.

[9] J. Arrillaga, N.R. Watson, Power System Harmonics, John Wiley \& Sons, Ltd, New Jersey, 2003.

[10] Cuk, V.; Nikolic, A.; Zigic, A., "A Development System for Testing Integrated Circuits Used for Power and Energy Measurements," in Proceedings of 13th International Power Electronics and Motion Control Conference EPE-PEMC 2008, pp. 1426-1431, Poznan, Poland, September 2008.

[11] N. Kehtarnavaz, S. Mahotra, Digital Signal Processing Laboratory: LabVIEW-Based FPGA Implementation, BrownWalker Press, Florida, 2010.

[12] National Instruments, Getting Started with LabVIEW, June 2010, Publication No. 373427G-01.

[13] Laskar, Shahedul Haque; Muhammad, Mohibullah, "Power Quality Monitoring by Virtual Instrumentation using LabVIEW," Proceedings of 2011 46th International Universities' Power Engineering Conference (UPEC), pp.1,6, 5-8 Sept. 2011.
[14] Power Quality Standard EN 50160:2010, Voltage characteristics of electricity supplied by public electricity networks, 2010

[15] Lee, R.P.K.; Lai, L.L.; Tse, N., "A Web-based multi-channel power quality monitoring system for a large network," Power System Management and Control, 2002. Fifth International Conference on (Conf. Publ. No. 488) , vol., no., pp. 112-117, 17-19 April 2002.

[16] Yue Fuchang; Shao Lin; Wu Zaijun, "Modeling and implementation of power quality monitoring device based on IEC 61850," 2012 China International Conference on Electricity Distribution (CICED), pp.1,5, 10-14 Sept. 2012.

[17] Wanfang Xu; Gang Xu; Zhijiang Xi; Chuanyong Zhang, "Distributed power quality monitoring system based on EtherCAT," Electricity Distribution (CICED), 2012 China International Conference on , vol., no., pp.1,5, 10-14 Sept. 2012.

[18] Yi Zhang; Honggeng Yang; Gong Cheng, "Application of 3G Technology in Power Quality Monitoring System," 2012 Asia-Pacific Power and Energy Engineering Conference (APPEEC), pp.1,4, 27-29 March 2012.

[19] Jiang Xian-kang; Ren Jian-wen; He Peng-yun, "Analysis and Application of Power Quality Data from Power Quality Monitoring Network," 2011 Asia-Pacific Power and Energy Engineering Conference (APPEEC), pp.1,4, 25-28 March 2011.

[20] Nikolic, A.; Naumovic-Vukovic, D.; Skundric, S.; Kovacevic, D.; Milenkovic, V., "Methods for power quality analysis according to EN 50160," in Proceedings of the 9th International Conference Electrical Power Quality and Utilization EPQU 2007, pp. 1-6, Barcelona, Spain, October 2007.

[21] Nikolic, A.; Babic, B.; Zigic, A.; Miladinovic, N.; Milosavljevic, S., "Multi-channel System for Remote Power Quality Monitoring of Electricity Supplied by Public Distribution Networks," in Proceedings of the XIX IMEKO TC-4 Symposium, pp. 1-6, Barcelona, Spain, July 2013. 